\title{
A new method of processing high-rate rocket telemetry data
}

\author{
Zhu Zebo, Chen Jian
}

China Satellite Maritime Tracking and Control Department, Jiangyin, 214431, China

Keywords: high-rate; telemetry data; distributed real-time system

\begin{abstract}
Aimed at the problem of the ability of high-rate rocket telemetry data process is limited, this article analyses the characteristic of telemetry data process and put forward an architecture of distributed process. And the keys of system are explained which contain telemetry data mapped, data fused and time adjusted. The prototype system is discussed that it is useful for the ability of process of the data node, and which is fulfill the requirement of advanced process ability.
\end{abstract}

\section{Introduction}

In recent years, new models of spacecraft increasing amount of data transfer, high bit-rate (10Mbit / s or more) instead of the previous low bit rate data transmission (2Mbit / s), and with the application of communication technology, constantly moving higher rate code development, providing technical support for flight test the accumulation of more data in the future. With the high rate of use of telemetry, real-time processing capability of the software put a higher demand.

For ground TT \& C systems, especially ground control center, launch segment tasks that require real-time monitoring of various spacecraft sensor status, flight attitude data, real-time processing of various types of parameters require not only state, drawing all kinds of curves, also you need to determine the various real-time instruction time, programmable instructions, etc. The ship is currently processing telemetry data center systems generally use hot standby to handle strategy, processing capacity is limited to a single server processing power. For low bit rate telemetry data processing, the server generally competent enough, but the face of high rate telemetry data (10Mbit/s, even $100 \mathrm{Mbit} / \mathrm{s}$ or more), an increase of stand-alone processing capacity can not meet the growth rate telemetry processing brings overhead.

Therefore, for this problem, the analysis of telemetry data processing characteristics, based on a real-time distributed processing ideas, telemetry data to multiple decomposition parallel processing on the server, you can improve the high rate telemetry data real-time processing capability, can improve the adaptability task full use of existing hardware and software resources.

\section{Telemetry Data Processing Features}

Telemetry is a parameter object to be measured from a distance, through experience, acquisition, via a transmission medium to the receiver demodulates and location recording, a measurement process. A combination of the above-enabled devices called telemetry system, which is Missiles (including launch vehicle), TT \& C system important part. By telemetry processing parameters, you can monitor the internal environment, the state of equipment and physiological state of the target aircraft during flight astronauts like, can also be analyzed by spacecraft telemetry data to evaluate performance, provide the basis for fault diagnosis and design improvements.

Because of the important role of spacecraft telemetry data in flight test, the telemetry data in real-time processing has the following characteristics:

a. Real-time high. For important events characterizing the flight parameters to determine or calculate immediately plotted, and then judge decided to implement the program the next flight or send remote commands based on parameters related to the state.

b. Multi-logic. Since the serial transmission of telemetry data, telemetry data processing time, the need to transfer the relationship between the order parameters, physical characteristics, flight status, time series and other factors considered, logical judgment, to get the final result.

c. Data and time correlation. Typical time instruction including jump instruction, instruction and 
digital position control instruction. Jump instruction before the current command generating means, the measured value remains unchanged, after the instruction occurs, the original level value jumps to another level values, including pulse and transition fixed transition two. Position instruction means that when instruction occurs, one or a few word changes telemetry. Digital instruction, in addition to including specialized instruction channel temperature measurement, but also includes the shutdown point parameters in computer word computer word processing and the like. Time processing of instructions only need to determine the appropriate time range, especially for channel multiplexing instructions, the same channel is allowed to occur repeatedly instruction judge the situation in different time frames, also needs to be judged according to the value of the data before and after the instruction.

\section{System Architecture Analysis and Design}

The ship is currently telemetry data processing center machine with full duplex symmetrical hot backup system, the system consists of two perfectly symmetrical control computer system through the duplexer modules, two systems running the same program at the same time, while receiving telemetry tracking ship sent by the device spacecraft telemetry and data processing, choose a system of external control module output data through the duplexer. This system, if one set of system failure or abnormal, the other can take over a system failure is normally run to ensure that the system does not stop working from time continuity.

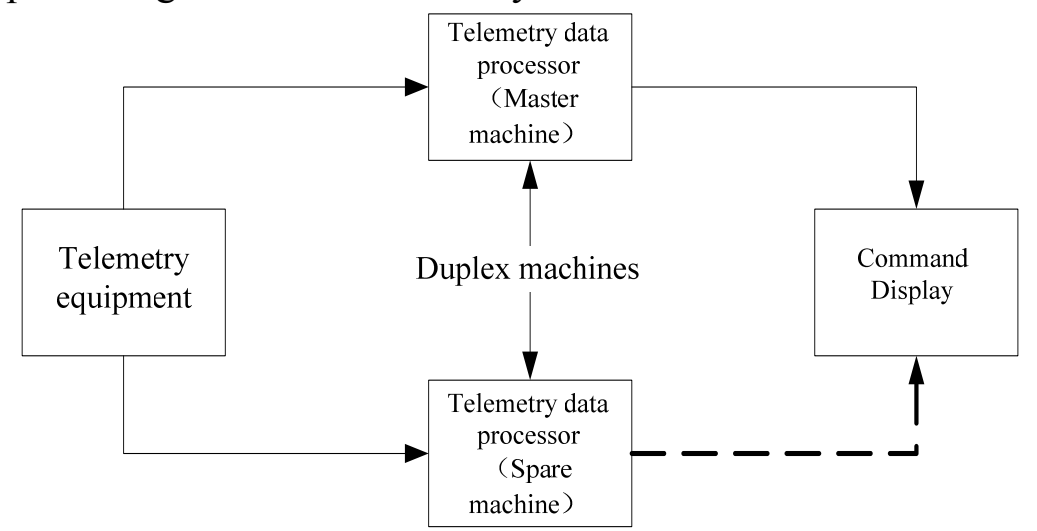

Figure 1. Duplex Hot Standby System Processing System

From the perspective of system operation, full duplex symmetrical hot backup system to ensure that the subsystem level of redundancy to improve reliability and maintain uninterrupted operation. But for processing power, its maximum processing capacity is still limited to the single largest processing capacity. For high rate telemetry data processing, the amount of data increases as the network data reception, stand-alone processing power, network data transmission capacity increases can not be unlimited, therefore, consider the telemetry processing tasks to multiple servers simultaneously exploded deal with.

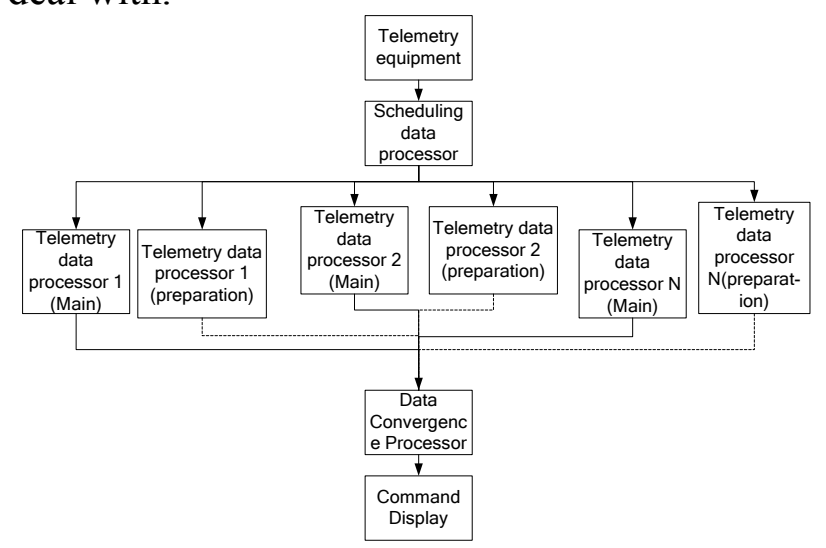

Figure 2. The distributed telemetry data processing

As can be seen from Figure 2, compared to the current telemetry data processing mechanism, the 
new telemetry data processing subsystem increases the processor scheduling, data aggregation processor, and may increase demand telemetry processor.

Scheduling data processor is mainly responsible for receiving telemetry equipment for telemetry data, and dispatch it to the appropriate processor handle and coordinate all data processing node operations. In order to improve the reliability of real-time telemetry data processing, real-time, in accordance with the respective data processing nodes running hot standby mode duplex data processing tasks simultaneously, when the primary node task fails, try using an alternate node data processing task result, if the standby node running the task fails, only the error message is logged and does not transfer to the data processor scheduling progress report, continue to the next data processing tasks.

Task scheduling data processor according to the model, to distinguish between different spacecraft mission, divide the data processing tasks are assigned to different lengths of data processing nodes.

\section{Key Techniques}

\subsection{Telemetry data decomposition}

For real-time telemetry data, it is a time-dependent serial data stream, commonly spacecraft telemetry data formats PCM telemetry, subcontracting telemetry, analog telemetry. Telemetry is a serial PCM stream in accordance with the hierarchy telemetry bits, bytes, and the frame format generated. Packet Telemetry is based on sub-way dynamic hierarchical data management, complete the multi-source multi-user programmable PCM telemetry data telemetry system.

Telemetry data in real-time processing, because of the time, it showed a time-dependent serial data stream. Data scheduling processor scheduling in the decomposition of the telemetry data using time-slice manner decomposition of the received telemetry data are transmitted to the different data processing nodes in parallel.

The telemetry data is broken down into a number of pieces of data, the processing means for each time slice compared to the entire data processing time is relatively small. Thus, parallel processing of each slice, and the slices are small pieces of data, then the process will have a better load balancing. The size of the fragment depends on the spacecraft telemetry format, code rate and data node processing capability. For the use of PCM Telemetry rocket telemetry data, its frame format full frame, sub-frame, sub-frame, each full frame contains a complete computer word, in the handling of parameters, the smallest full-frame processing unit . Scheduling data processor should be an integral multiple of a full frame length of telemetry data decomposition to the processing nodes, the nodes to improve processing efficiency.

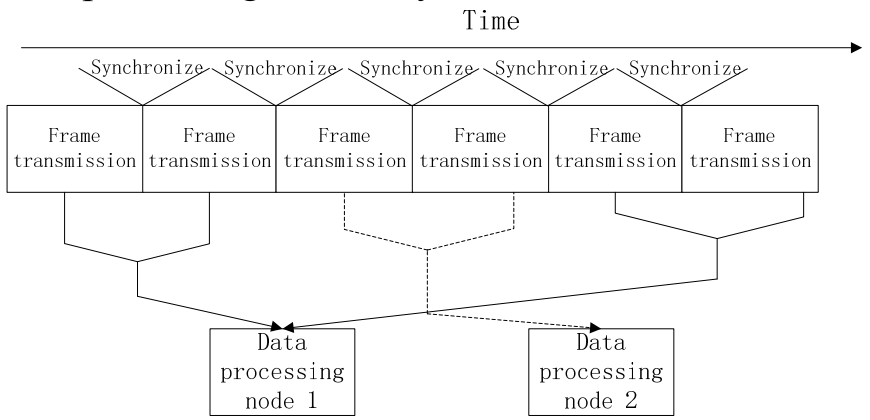

\subsection{Telemetry data fusion}

Figure 3. telemetry data decomposition

According to real-time telemetry data processing requirements, under normal circumstances, a variety of temperature, pressure, speed and other telemetry parameters need to draw one point per second for display use command, this requirement limits the processing time of real-time processing, the processing per second least a set of the data results.

For stand-alone use for processing telemetry data, there is no problem of data fusion, because the entire process is the serial data; for distributed processing mode, since the telemetry data is decomposed into a plurality of processing nodes in parallel, and the final result is attributed to the 
processing telemetry data for displaying a second point, therefore, the telemetry data processing results were different nodes key information integrated into distributed processing.

Information fusion is a key technology of distributed processing, integration means there are centralized fusion, autonomous integration, hybrid fusion and integration of four multi-stage structure. Considering the telemetry data processing features, mainly hierarchical integration.

Fusion hierarchical structure characteristic is that each node has a data processing, and the telemetry data obtained in this node to process local produce results, then the results sent to the node data fusion, data fusion node based on each processing node telemetry local middle results complete integration of telemetry data.

Rocket telemetry parameters can be divided into the assist level parameters, twelve parameters, three parameters + level above parameters? ; Also be divided into dynamic system parameters, control system parameters, the overall system parameters. Parametric change frequency telemetry parameters can be divided into fast and slow varying parameters varying parameter, the parameter is a random variable speed parameter, it can not be described by explicit data relationships, and can only be used to describe the probability and statistical functions, slowly varying parameters are determined data, mathematical relationships can be explicitly described, the exact value can be obtained at any time.

In addition to the basic characteristic parameters, there are certain parameters specific variation: The axial overload, power system pressure and level with the time history of flight, each feature segment ignition, off, off point will be significantly variation, as well as the associated characteristic parameters, such as command parameters and ignition, shutdown, separation of closely related actions; voltage control system, current and servo systems, attitude control engines and other associated parameters of the operation and has had strict order.

\subsection{Time Calibration}

For distributed processing telemetry data, an important prerequisite for a multi-node data processing result information fusion is the time of each node must be strictly unified representation.

Currently the main way to solve the problem when the system has two: one is the use of high-precision satellite positioning system clock; the other is the individual nodes are high stability rubidium atomic clock as a synchronizing signal. Considering tracking ship environment, using high-precision clock satellite positioning system as a system reference signal, each node receives a unified time system clock signal by extending multi-function control card, in the processing of data by reading the multifunction control card time to achieve a unified time calibration signal.

\section{Summary}

With telemetry data rates increase, tracking ship Centers telemetry subsystem facing single real-time processing performance bottlenecks. This paper presents a distributed system architecture, high rate telemetry data to improve real-time processing capability, through the decomposition processing telemetry data processing and integration, improve system scalability, the ability to take full advantage of the processing node processing, to meet the growing demand for processing power .

\section{Reference}

[1] Glavieux B C.A Near Optimum Error Correcting Coding and Decoding:Turbo Codes[J].IEEE Transaction on Communication.2012,44(10)

[2] CCSDS 131.0-B-1-2013.TM Synchronization and ChannelCoding[S].Washington D C:CCSDS Secretariat,2013

[3] Cousineau,Denis,Fitting the three-parameter weibull distribution: Review and evaluation of existing and new methods. Optik, 2009, 120(3):127-13. 